\title{
Vegetation cover analysis using a low budget hyperspectral proximal sensing system
}

\author{
Antonio Cenedese $\left({ }^{1}\right)$, Massimo Miozzi $\left({ }^{1}\right)$, Alvise Benetazzo $\left({ }^{1}\right)$, Alessandro Paglialunga $\left({ }^{2}\right)$, \\ Carlo Daquino $\left({ }^{3}\right)$ and Roberto Mussapi $\left({ }^{3}\right)$ \\ (1) Dipartimento di Idraulica, Trasporti e Strade (DITS), \\ Università degli Studi di Roma «La Sapienza», Roma, Italy \\ ${ }^{(2)}$ Superelectric sas, Tempio Pausania (SS), Italy \\ $\left(^{3}\right)$ Agenzia per la Protezione dell'Ambiente e i Servizi Tecnici (APAT), Roma, Italy
}

\begin{abstract}
This report describes the implementation of a hyperspectral proximal sensing low-budget acquisition system and its application to the detection of terrestrian vegetation cover anomalies in sites of high environmental quality. Anomalies can be due to stress for lack of water and/or pollution phenomena and weed presence in agricultural fields. The hyperspectral cube (90-bands ranging from 450 to $900 \mathrm{~nm}$ ) was acquired from the hill near Segni (RM), approximately $500 \mathrm{~m}$ far from the target, by means of electronically tunable filters and 8 bit CCD cameras. Spectral libraries were built using both endmember identification method and extraction of centroids of the clusters obtained from a $k$-means analysis of the image itself. Two classification methods were applied on the hyperspectral cube: Spectral Angle Mapper (hard) and Mixed Tuned Matching Filters (MTMF). Results show the good capability of the system in detecting areas with an arboreal, shrub or leafage cover, distinguishing between zones with different spectral response. Better results were obtained using spectral library originated by the $k$-means method. The detected anomalies not correlated to seasonal phenomena suggest a ground true analysis to identify their origin.
\end{abstract}

Key words hyperspectral proximal sensing - tunable filters - vegetation classification

\section{Introduction}

A hyperspectral passive remote sensing technique is applied to measure and analyze the reflection of the land surface as a result of solar radiation. The object is framed by selecting and moving a narrow window of the electromagnetic

Mailing address: Dr. Massimo Miozzi, Dipartimento di Idraulica, Trasporti e Strade (DITS), Università degli Studi di Roma «La Sapienza», Via E. Stilone 20, 00174 Roma, Italy; e-mail: m.miozzi@tin.it radiation within the spectral interval of interest. The luminous intensity values in every single point (pixel) on all images acquired in the visible and near-infrared range represent the reflectance spectrum of the objects framed. Such information allows a territorial classification based on the reflectance characteristics of the materials constituting its surface to be carried out.

The introduction of a low budget proximal sensing level for hyperspectral analysis provides an innovative instrument for monitoring zones of environmental importance and areas for agricultural use. The simple utilization and low operating costs facilitate the possibility of a systematic monitoring in space and time of the parameters observed. The small-medium spatial scale which the proximal sensing refers to, allows us both to deepen analysis up to very high 
detailed levels (both in space and in radiation spectrum) and to supply useful spectral information for validation and calibration of instruments operating on a larger spatial scale or with simpler multispectral resolution, as the equipment mounted on airplanes or satellites. At present, the system described herein is placed at the ground level on a rise located near the territory (scene) to be analysed.

The present contribution provides general information on the three main tasks investigated within the research. Section 2 describes the «low budget» technology (system domain); Section 3 provides an overview of the algorithms used for preprocessing and classifying images (data domain); Section 4 briefly presents a review of the relations among vegetation, its state of health and spectral reflectance, and Section 5 reports some of the results (information domain).

\section{The «low budget» system domain}

The aim of the hardware architecture is to realize a hyperspectral image acquisition system able to capture images of a target, which is 300$500 \mathrm{~m}$ far from the measuring station (placed at ground level on a hill), using video cameras and birefringent filters placed on the camera optics.

In the present work two Varispec (CRI, Cambridge Research \& Instrumentation, Inc.) filters were used. They are based on the same physical criteria, consisting in multistrate birefringent liquid crystal filters, and work on two different operative ranges: $400-700 \mathrm{~nm}$ (VIS filter) and 650$1050 \mathrm{~nm}$ (NIR filter) respectively.

The main property of the filters consists in their capability of being tuned on a wavelength within their operative range. The passing band obtained can be described using a Gaussian distribution with a standard deviation of approximately $5 \mathrm{~nm}$. The electromagnetic radiation spectrum scanning, which is guided by a synchronization device, is performed using a $5 \mathrm{~nm}$ step: in this way a 90 band hyperspectral cube is obtained, ranging from $450 \mathrm{~nm}$ e $900 \mathrm{~nm}$.

The filters are mounted on two 8 bit, $576 \times$ $\times 720$ pixel as spatial resolution CCD cameras SONY XC-8500CE. Due to the quantum effi- ciency of the camera's CCD (Sony Corporation, 1996), a range smaller than the maximum one (450-900 versus $400-1050 \mathrm{~nm}$ ) is chosen.

A magnetic support in DV-PAL standard $(25 \mathrm{~Hz})$ allows the acquisition of the video stream from the cameras. The passing band of the Varispec filter is moved once per second. This delay time was chosen for two reasons: the filter needs a maximum of $90 \mathrm{~ms}$ in its worst performance and about $30 \mathrm{~ms}$ in mean to tune itself on a preselected band; besides, if the filter is kept for $1 \mathrm{~s}$ on a given wavelength, about 25 images can be acquired at the same passing band. Those images will be used to validate the data and to apply preprocessing techniques to improve image quality.

A schematic diagram of the acquisition system (two channels, one for each filter) is plotted in fig. 1 .

\subsection{Raw data extraction}

After the image digitization (via IEEE1394), different passing bands were detected evaluating the mean value of each image inside the video stream: the same passing band corresponds to the same mean value.

Figure 2 shows the behavior of the mean intensity value versus the filter-passing band (image sequence in video stream): the transition of the filter from a wavelength $\left(\lambda_{i}\right)$ to the successive $\left(\lambda_{i+1}\right)$ give rise to instability in the intensity mean value. After a small number of images, the mean intensity values become stable (and they may be the correct ones). This number of images changes from band to band, but it always remains very small.

With the aim of obtaining a more consistent set of data, the operator has to choose a set of stabilized images belonging to a specific band (one set per each band).

A Proper Orthogonal Decomposition (POD) is applied to each set of images: the most energetic mode is used to apply the revert algorithm (Ready and Wints, 1973). In this way, most correlated data are retained in the reprojected image, which can be assigned to the corresponding wavelength as a representation of the land surface absolute reflectance. 


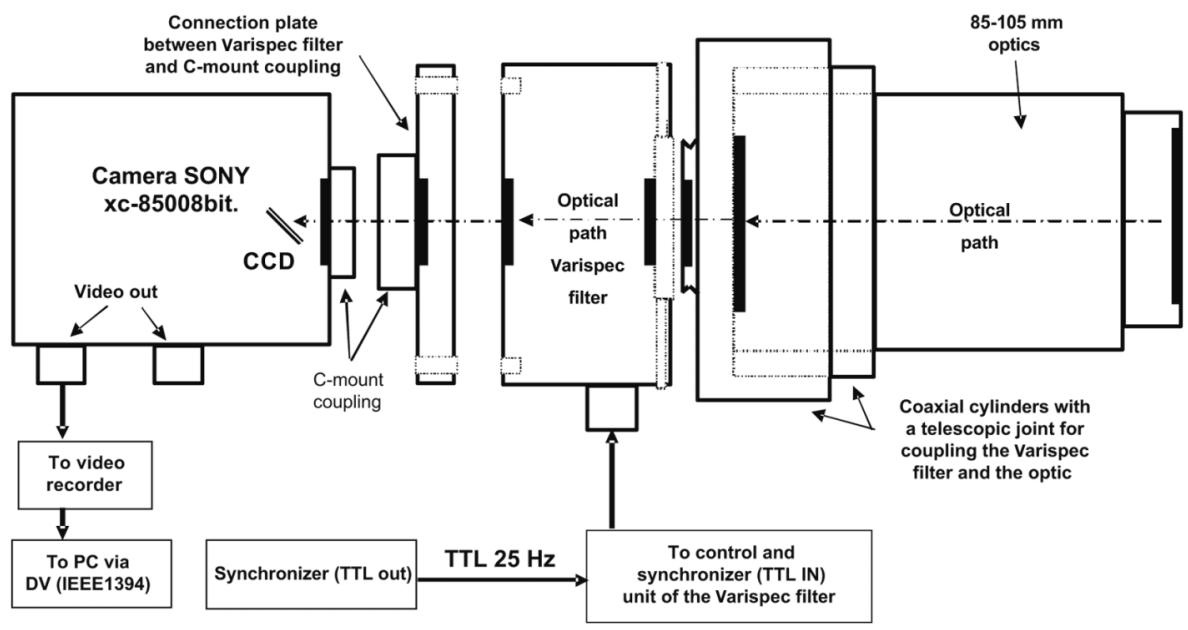

Fig. 1. The acquisition system.

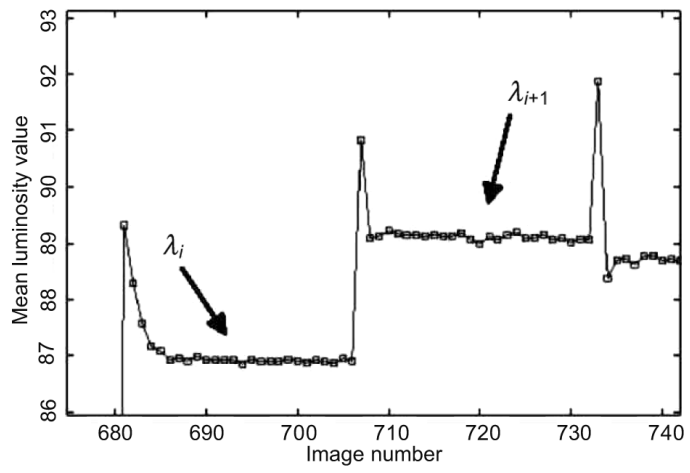

Fig. 2. Mean value of the image intensities.

\section{Data analysis}

\subsection{Preprocessing}

The system domain results (video stream) were preprocessed to lead back the data to the spectrum-normalized values in the same spatial context (warping).

The need to extend the field of spectral surveying beyond the visible range required the use of two tunable filters operating on two different regions of the electromagnetic radiation spectrum. To this aim the equipment available requires two filters to be used: they operate simultaneously from two different positions on twin instrumentation. In this case, the images of the same object differ from each other for spins, translations, scale and angular sliding.

The construction of the hyperspectral cube needs an affine transformation (warp). A set of corresponding points on two images is located (manually or employing optical flow techniques) and the coefficients of the transformation are kept. After warping (NIR images are warped on the basis of the VIS image) the operator selects the spatial window the two intermediate cubes have in common and the final cube is constructed by superposition of these two intermediate ones.

Finally, in order to operate in terms of relative reflectance the IARR calibration algorithm (Internal Average Relative Reflectance) was applied.

\subsection{The classification process}

The process of image classification, in the classical (hard) sense, consists in assigning each pixel to a class with common characters (Campbell, 1996). Soft approaches introduce a 
«degree of membership» of each pixel to each class. This process is schematized in three successive steps: the extraction of the meaningful data, the building of a spectral library and the labeling of the pixels (in a hard or soft fashion). The extraction of the meaningful data from the large (and redundant in spatial and spectral spaces) amount of information contained in the hyperspectral cubes, by means of spatial and spectral operators (i.e. Pixel Purity Index and Maximum Noise Fraction), simplifies the set of data using the same original information content. The identification of the pixels able to train the classificator to distinguish between different classes and to assign a label to each pixel drives the building of the spectral library. Those pixels are named endmembers: they are representative of «most pure materials» inside the scene. It is necessary to identify a discriminant function to separate one class from another. This step can be supervised or unsupervised. The use of a discriminant function permits us to label the pixels belonging to the whole image. The result of the process is a map with a label per each pixel (in the hard case), or a collection of $N$ maps (one per each class) with the membership values per each pixel.

\subsubsection{Endmember method}

The endmember method applied consists in the identification of a limited set of pixels, named endmembers, that can be regarded as «pure materials» inside the scene. The signature of those pixels is used to model the reflectance spectrum at each pixel of the original image, in a linear combination of a small number of endmembers. The partially automated method used within this work consists in a reduction of the complexity in both the spectral (MNF, Green et al., 1988) and the spatial (PPI, Boardman et al., 1995) domain. The set of data obtained is plotted in an $n$-dimensional visualizer. To obtain endmember spectral signature, pixels with similar spectral characters are grouped together by the operator: this operation gives good results because endmembers fall on cusped of the $n$-dimensional convex hull. The spectral library is used to classify the image.

\subsection{2. $K$-means method}

$K$-means algorithm (Duda and Hart, 1973) is a versatile tool extensively applied in clustering problems. It is often used to produce a start set of spectral signatures that can be used in more complex analysis. The aim of the method is to group data as a function of a distance (in this case Euclidean) from the centroids of the various clusters.

The algorithm first needs the number $N$ of clusters to be chosen. This is a crucial point, since no analytical methods are provided to estimate this parameter. This task could be achieved using the ISODATA algorithm, to be implemented in further developments. As a second step, $N$ arbitrary cluster centroids are chosen. The remaining pixels are then assigned to the cluster with the minimum Euclidean distance from the centroid. The new centroid position is evaluated using the pixels belonging to its cluster and a new classification step is performed until the centroids of the clusters keep an almost fixed position. Results are very stable and do not depend on the initial choice of the centroids.

\subsubsection{Classifying the image}

In order to label the pixels in the image, the results obtained using the Mixed Tuned Matching Filter (MTMF) technique (Harsanyi and Chang, 1994) are shown. In this way a matched filter score for each pixel is obtained, together with an «Infeasibility» degree. This latter value can be used to control the number of «false positive» that are sometimes found using MTMF (RSI, 2001).

\section{Hyperspectral analysis for detection of vegetation stress}

The peculiarities of the amount of the solar electromagnetic radiation reflected by vegetation depend on the biochemical reactions occurring within the leaf and its physiological characteristics (Kumar et al., 2001). These characteristics include the leaf's anatomy (thickness and structure of the mesophyll), pigments and other bio- 


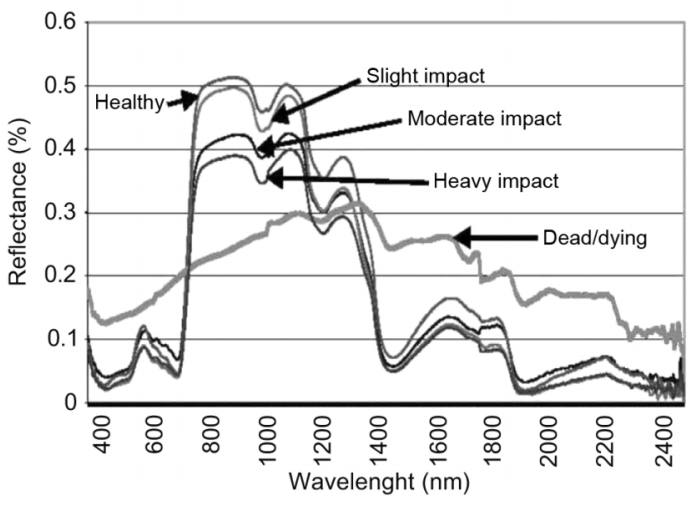

Fig. 3. Vegetation reflectance curves.

chemical constituents (chlorophyll, carotenoids, proteins, lignin), the physiological state represented by the water content, the photosynthetic efficiency and the fluorescence of chlorophyll and other molecules.

A typical spectrum of pigments absorption characterizes a condition of healthy vegetation (fig. 3). The stress degree is associated with the reduction of the chlorophyll content, with an increase of reflectance in correspondence to the peak on the green wavelength and on the chlorophyll well (wavelength around red), a break of the leaf's cells, with a reflectance reduction in the NIR plateau (fig. 3), until death (fig. 3).

Three radiation response regions can be identified.

The visible region range (400 nm-720 nm) includes the wavelengths activating the main biological systems regulated by solar radiation. The carbon reduction (photosynthesis) by means of the solar radiation takes place inside the leaves' cells, due to its biochemical components. The wavelengths of the visible region are selectively absorbed from pigments. The visible reflectance, particularly in the green portion of the electromagnetic spectrum, in proximity of $550 \mathrm{~nm}$, and in the red portion, in proximity of $710 \mathrm{~nm}$, considerably increases as a result of different stress conditions and for various vegetable species (Carter, 1993).

The red-edge range $(690 \mathrm{~nm}-720 \mathrm{~nm})$ regards the spectrum region characterized by a low red chlorophyll reflectance (chlorophyll well) to a high reflectance around $800 \mathrm{~nm}$ (NIR plateau), associated with leaf internal structure and water content. The position of the inflection, called REIP (Red Edge Inflection Point), varies among different vegetable species, however it is contained inside the range $680 \mathrm{~nm}-750$ $\mathrm{nm}$. A shift of the inflection towards smaller wavelengths (red edge blue shift) is a warning sign of vegetation stress.

Plants generally have a large reflectance and transmittance in the near-infrared region (700 $\mathrm{nm}-1300 \mathrm{~nm}$ ). In contrast to the energy of the light in the visible wavelengths, the energy levels of near-infrared light are not large enough for photochemical reactions to take place and chloroplasts and other pigments do not absorb the radiation. The passage of light within the leaves is a direct consequence of the shape and size of leaf cells and the spatial distribution of empty spaces among them. The near-infrared reflectance does not change under stress conditions or it may slightly change. The effects shown are a consequence of the reduction of the water content in the leaves; dehydration effects are observed around $1400 \mathrm{~nm}, 1900 \mathrm{~nm}, 2000$ $\mathrm{nm}$ and $2400 \mathrm{~nm}$ (Carter, 1993).

\section{Results}

Results presented here concern a data acquisition performed near Segni (Rome, Italy); the investigation focused on the rural environment in the valley, down to the village. Two different scenes are illustrated here to show the capabilities of the technique.

In the framework of the scenes, meadowlands, sowed fields, shrubs and man made manufactures can be identified (figs. 4 and 5). Different land covers can be distinguished using their spectral characters and their space locations.

Pixel dimension at ground is about $25 \mathrm{~cm}$.

The clusters obtained with the unsupervised technique have been gathered together in groups that take into account the spatial position of classified pixels belonging to the main categories in the framework: meadow lands, sowed fields, leaf vegetation etc. Inside those 


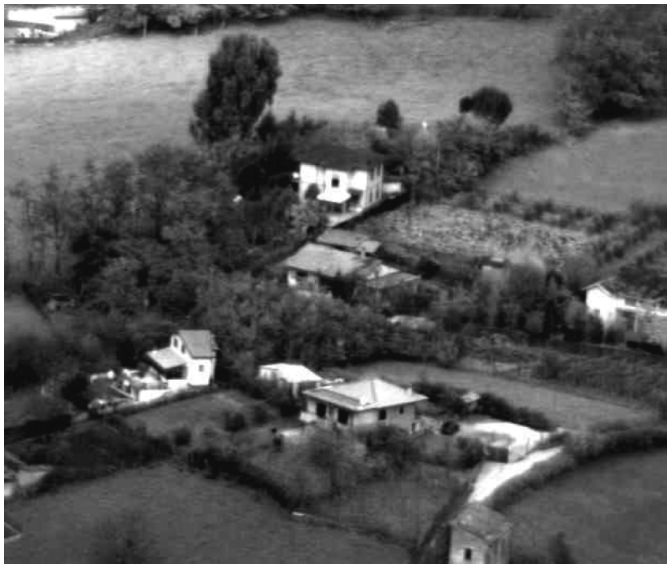

Fig. 4. Scene 1.

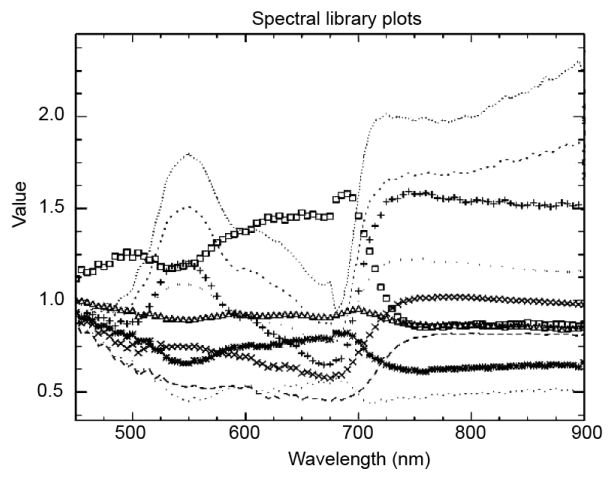

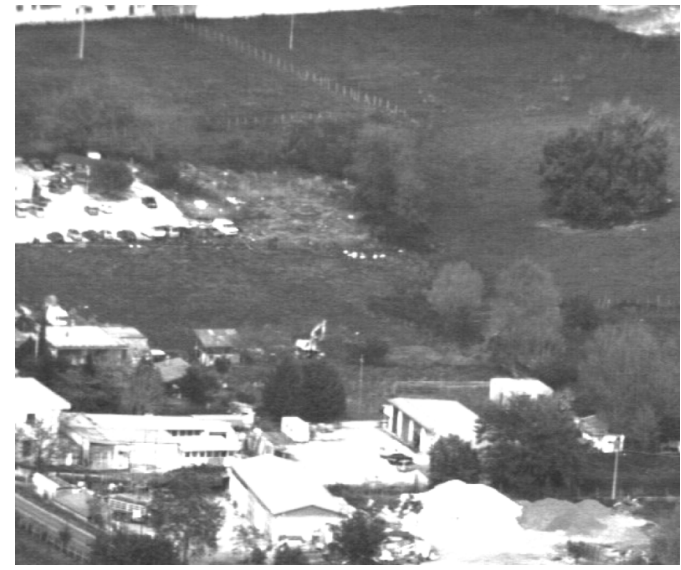

Fig. 5. Scene 2 .

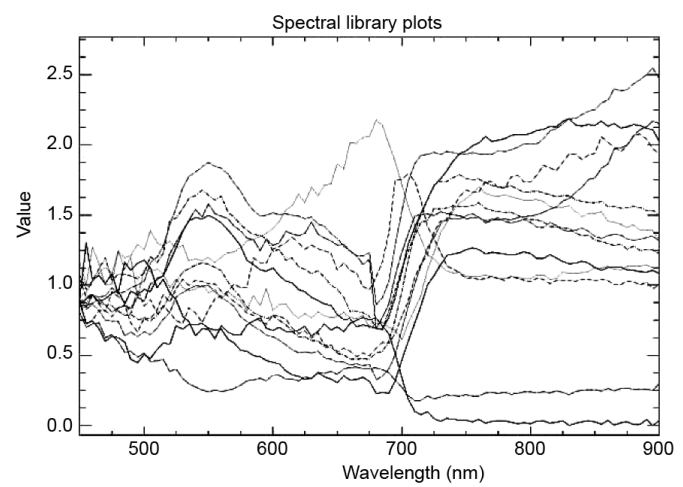

Fig. 6. Spectral signatures (clusters «Meadows» and «Shrubs»). K-means (left) and endmemebers (right).

groups, clusters with analogous spectral characters have been merged together. This «paesistic» choice has been made because one of the targets of this research was to classify the land covers in a smaller number of different signatures. As a different approach, a «pixel» choice could have been made, to deeply analyze linear mixing inside each pixel.

\subsection{Scene 1}

The spectral libraries obtained with the partially supervised (endmember) and unsuper- vised ( $k$-means) techniques are compared. Figure 6 (left) displaces the centroids of the clusters related to meadow lands («Meadow») and shrubs («Shrub»), extracted with a $K$-means analysis.

The spectral behavior is in a good agreement with the expected results: the vegetation reflectance trend shows the typical «peaks and throats» shape. The spectral signatures of the same scene, obtained using a partially unsupervised technique, show a similar behavior for vegetation and shrubs (fig. 6 , right), but with a larger noise level: this is a symptom of the greater uncertainty in the classification process. 


\subsection{Scene 2}

The spectral signatures for «Meadow» species shown in fig. 7 are the result of the unsupervised classification process.

In this plot, an anomalous behavior of the category «Meadow 6», that was spatially associated to the vegetation group, is clearly observed. This behavior can be emphasized merging together in the «Meadow» spectrum clusters related to «Meadow 1», «Meadow 2», «Meadow 3»,

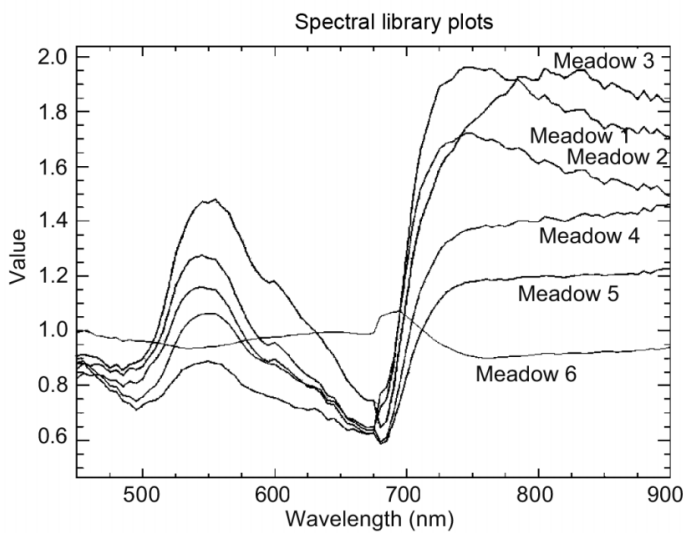

Fig. 7. Centroids of the «Meadows» clusters.

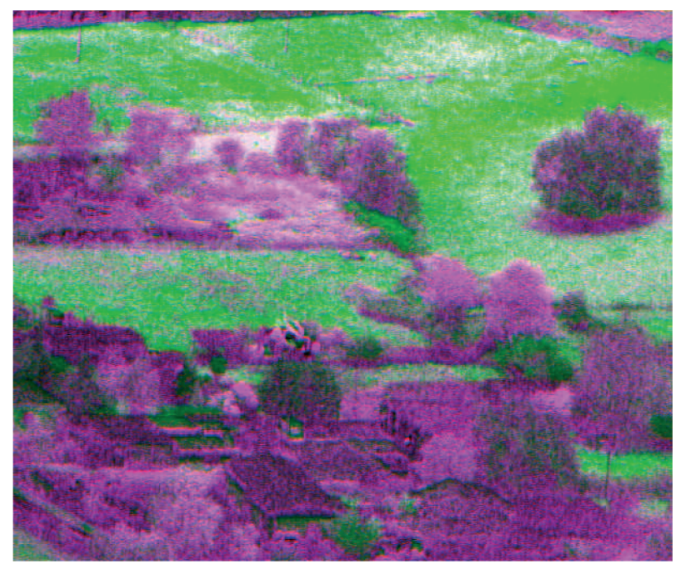

Fig. 8. False colors: «Meadow $6 »(\mathrm{R}+\mathrm{B})$ and «Meadow $1 / 2 / 3 / 4 / 5 »(G)$.
«Meadow 4» and «Meadow 5». A new spectral library was produced, containing «Meadow 6» and this new spectral signature.

This library was applied inside a targeting algorithm: results are reported in fig. 8. In this image, plotted with false colors, the classification capability of the system can be shown.

The green buffer of the image was filled using targeting results (matched spectrum scores from MTMF) from «Meadow» spectrum; red and blue buffers were filled using «Meadow 6». Grassy materials are very well emphasized inside the image: they are clearly different than the vegetal part of trees and shrubs. «Meadow 6» spectrum, on the contrary, is mainly concentrated in the center of the image, in the field near the parking lot.

The characteristics of the spectral signature «Meadow 6» do not permit any hypothesis on the nature of the land cover material: this area, from the standpoint of the present work, has to be classified as a «Paesistic Anomaly» and should be submitted to a ground truth investigation in the framework of monitoring actions.

\section{Conclusions}

The implementation of a proximal sensing hyperspectral image acquisition system has been described in this work, together with the main results obtained in applying the system itself to the test study of a real case.

The main items of this system are its low budget implementation, its portability and its simple use. The transfer of this hardware architecture on an airborne platform (balloon or helicopter) will permit the execution of proximal sensing measures also when good ground points of observation are lacking.

More data specific items concern the availability of a robust set of hyperspectral images, obtained after robust POD filtering of data acquired at the same wavelength. An improvement of the quality of the results will be gained avoiding the data recording on magnetic tape, which at present imposes DV-PAL compression. A frame grabber able to acquire uncompressed grey scale images at full size will be used to perform the digitisation process. 
In this implementation of the system, CCD video camera quantum efficiency represents a limit in the amplitude of the investigable range: signal to noise ratio at wavelengths lower than $450 \mathrm{~nm}$ and higher than $900 \mathrm{~nm}$ is very low. The substitution of the sensor technology for the NIR channel of acquisition has been planned for the future.

Applications of this proximal sensing hyperspectral system range from environmental investigations about stress conditions in vegetation land cover, due to lack of water and/or pollution phenomena (i.e. unauthorized landfill disposals), to the analysis of the coastal zones to detect the presence and the relevance of algae populations to prevent and control eutrophization phenomena, to the investigation of the river neighbours to detect unauthorized discharges or anomalies in the riparian vegetation and as a support in the precision farming activities.

The low budget and the simple use of the system makes it interesting also for small public administrations that are interested in developing an effective control of urban development and rural areas assessment.

Moreover, the proximal sensing approach could constitute a salient source of data in calibrating data from more remote and sophisticated acquisition platforms.

\section{REFERENCES}

Arrigoni, O. (1973): Botanica Generale (Casa Editrice Ambrosiana, Milano), pp. 541.

Boardman, J.W., F.A. KruSE and R.O. Green (1995): Mapping target signatures via partial unmixing of AVIRIS data, in Summaries of the V JPL Airborne Earth Science Worlshop, Pasadena, CA.

Campbell, J.B. (1996): Introduction to Remote Sensing (Taylor \& Francis Ltd, London), 2nd edition.

CARTER, G.A. (1993): Responses of leaf spectral reflectance to plant stress, Am. J. Botany, 80 (3), 239-243.

DudA, R.D. and P.E. HART (1973): Pattern Classification and Scene Analysis (John Wiley \& Sons, New York), pp. 482.

Green, A.A., M. Berman, P. Switzer and M.D. Craig (1988): A transformation for ordering multispectral data in terms of image quality with implications for noise removal, IEEE Trans. Geosci. Remote Sensing, 26 (1), 65-74.

HARSANYI, J.C. and C. CHANG (1994): Hyperspectral image Classification and dimensionality reduction: an Orthogonal subspace projection approach, IEEE Trans. Geosci. Remote Sensing, 32 (4), 779-785.

Kumar, L., K.S. Schmidt, S. Dury and A.K. SKIDmore (2001): Review of hyperspectral remote sensing and vegetation science, in Hyperspectral Remote sensing, edited by F. van Der MEER (Kluwer Academic Press, Dordrecht).

READY, P.J. and P.A. WINTS (1973): Information extraction, SNR improvement and data compression in multispectral imagery, IEEE Trans. Commun., COM-21, 10, 1123-1131.

RSI (2001): ENVI User's Guide (Research Systems, Inc.), vol. 1, pp. 435.

Sony CoRPORATION (1996): Sony XC-8500 CE Black-andWhite Camera Module Operating Instructions (Sony Ed.), pp. 86. 